\title{
Towards Quantifying Magnitude of Political Bias in News Articles Using a Novel Annotation Schema
}

\author{
Lalitha Kameswari, Radhika Mamidi \\ Language Technologies Research Centre \\ IIIT Hyderabad \\ Telangana, India \\ v.a.lalitha@research.iiit.ac.in \\ radhika.mamidieiiit.ac.in
}

\begin{abstract}
Media bias is a predominant phenomenon present in most forms of print and electronic media such as news articles, blogs, tweets, etc. Since media plays a pivotal role in shaping public opinion towards political happenings, both political parties and media houses often use such sources as outlets to propagate their own prejudices to the public. There has been some research on detecting political bias in news articles. However, none of it attempts to analyse the nature of bias or quantify the magnitude of the bias in a given text.

This paper presents a political bias annotated corpus viz. PoBiCo-21, which is annotated using a schema specifically designed with 10 labels to capture various techniques used to create political bias in news. We create a ranking of these techniques based on their contribution to bias. After validating the ranking, we propose methods to use it to quantify the magnitude of bias in political news articles.
\end{abstract}

\section{Introduction}

An increase in Internet and social media access has made it easier for people to know the happenings from all parts of the world. This improved reach of the media has led to people and organisations utilising such platforms to increase their popularity. With this extensive usage of mass communication, there is an increased risk of misinformation, fake news, twisting of facts, and the spread of controversies. In such cases, it is the responsibility of the media houses to verify the authenticity of the reported information and ensure that it is complete, neutral, and not misleading.

However, we often see instances where some news articles emphasize more on particular viewpoints selectively. In some cases, journalists and media houses either intentionally or subconsciously present biased information aligned with their own political ideologies. This can alter public opinion hugely and impact the decision of the general public. This phenomenon of political bias is very prevalent in democracies, especially during elections, where influencing public opinion can have far-reaching consequences.

Detection of political bias in the news is a complex task when it comes to a multi-party political system like India, where multiple parties operate at both state and national levels ${ }^{1}$. There has been limited work to date in this area, as discussed in Section 2. However, in a scenario where most of the political content contains bias in some form, it is not just sufficient to identify the existence of bias.

Therefore, we propose two important contributions through this paper - a fine-grained annotation schema specifically designed for analysing political bias and a new sentiment analysis based approach to quantify the magnitude of political bias in an article. We also present PoBiCo-21, an annotated corpus for political bias, containing 500 news articles along with their headlines annotated for various aspects of political bias using our novel annotation schema as discussed in Section 3. The dataset contains news articles in Telugu ${ }^{2}$, which is a low resource Dravidian language spoken primarily in the states of Telangana and Andhra Pradesh in India.

\section{Related Work}

The area of political discourse analysis is closely associated with media studies, political science, anthropology, sentiment analysis, and opinion mining. However, most of the work done is limited to English, primarily due to the high availability of data. For example, Jiang and Argamon (2008) identify

\footnotetext{
${ }^{1}$ https://bit. ly/3p74aHx

${ }^{2}$ https://en.wikipedia.org/wiki/Telugu_ language
} 
subjective sentences in blog posts to understand the political orientation of a given text. Nasab and Dowlatabadi (2015) proposed a rule-based linguistic method to identify bias in a news article based on the semantics of the headline and the article.

Gangula et al. (2019) and Cruz et al. (2020) used attention mechanism on different kinds of document representations to identify bias in an article. Kameswari et al. (2020) proposed a hybrid approach to improve the performance of such ML models by establishing a correlation between presupposition and bias.

Fan et al. (2019) pointed out that restricting to lexical level for detecting bias might not be sufficient and put forward a method of identifying spans of bias by looking at sentences/clauses which are speculative or tangential to the main point being talked about.

Zhou et al. (2011) made use of the comments and opinions of readers in order to classify articles as liberal or conservative. But the limitation to this approach is that it cannot perform well in a political setting where multiple alliances and several parties operate at different levels of the government.

All the work discussed above deals only with the detection of bias, and none of these approaches give us any further insight into the nature or the magnitude of bias in a given text. Our work fills this gap by creating a specialised fine-grained annotation schema for analysing and quantifying the political bias in a given text.

\section{Data and Annotation}

Our first requirement was a dataset of political news articles containing positive and negative bias along with some unbiased articles. For this purpose, we used the public dataset created by Gangula et al. (2019) as our primary data to begin with. Our aim is to annotate the dataset with different techniques through which bias is generated, and identify the levels of reporting at which it happens. This will be discussed in detail in Section 3.1. We removed the articles which were very short, or had no clear target of bias, either positive or negative. The statistics of our dataset are presented in Table 1.

The reason for relatively fewer unbiased articles in the corpus is due to the ubiquitous existence of some level of bias in most political articles. This can happen because of the affiliation of media houses with political parties - such as the Sakshi ${ }^{3}$

\footnotetext{
${ }^{3}$ https://en.wikipedia.org/wiki/Sakshi_
}

Telugu newspaper owned by the family of the Y.S. Jagan, the leader of YSRCP party and current Chief Minister of Andhra Pradesh. Other possible reasons include the incorporation of subjectivity, or the influence of the personal ideologies of the journalists, editors, and writers of an article, despite the ideal expectation of objective reporting.

\begin{tabular}{ll}
\hline Polarity of Bias & Number of Articles \\
\hline Positive & 180 \\
Negative & 220 \\
Unbiased & 100 \\
\hline Total & $\mathbf{5 0 0}$ \\
\hline
\end{tabular}

Table 1: Statistics of our dataset

\subsection{Political Bias Techniques}

Bias can be incorporated in news articles in several ways. It ranges from selection or gatekeeping bias, which is the selection and filtering of news broadcasted by media houses, since the set of world events is too large to be treated exhaustively. In the case of politics, the scope for subjectivity in selecting information about some specific event, person, or political party often induces bias due to resource constraints, editorial guidelines, ideological affinities, or even the fragmented nature of the information at a journalist's disposal (Bourgeois et al., 2019).

In this paper, we focus primarily on Presentation Bias which shows how the way of presenting some information can influence the readers directly or indirectly. Since the objective of introducing bias is to persuade the reader towards or against an entity, the methods used to create such bias can also be considered as propaganda. Political Scientist Harold Laswell defines propaganda as "the expression of opinions or actions carried out deliberately to influence the opinions or actions of individuals or groups for predetermined ends and through psychological manipulations". Herman and Chomsky (2010) came up with a theory on propaganda which views mass media as a group of businesses whose job is to sell readers and audience as a product to other advertising businesses primarily controlled by the government and the corporate sectors.

Based on the strong correlation between propaganda and bias, we used the list of all propaganda

(newspaper) 
techniques compiled from Propaganda and Mass Persuasion: A Historical Encyclopedia, 1500 to the Present by Grandstaff (2006). The list is available on Wikipedia ${ }^{4}$. The original list contains 68 techniques of propaganda. Out of these, many of them were not applicable to political bias. We removed such techniques and clubbed some very similar techniques in the context of political bias to finally create a list of 10 techniques discussed below. It is important to note that these techniques are not mutually exclusive - a given sentence/article may contain multiple techniques together.

1. Cultural/Identity Bias: This kind of bias includes all sorts of prejudices towards the class and the identity of a politician/party based on several divisions. Some examples are a subtle or direct portrayal of discriminatory attitudes like racism, casteism, ageism, sexism, etc., which could either be used positively or negatively to create bias.

2. Amplification: This is a term used to refer to the introduction of bias by overly hyping or exaggerating information about events/people/parties in order to present a skewed impression to the readers. It is often identified by the use of loaded language in political news. It can cause misrepresentation of the truth of an event or alter the reader's image of a politician/party/political event.

3. Personal Targeting: This technique is said be used when an individual is attacked/glorified for his personal traits instead of their political contributions. This is often done as an attempt to divert attention from other issues, or sometimes even as a way to show agreement/disagreement towards some policies or situations.

Ad Hominem or Personal attack is a technique of attacking some individuals or their political parties instead of attacking their arguments and policies. This can include name calling, stereotyping, labelling and scapegoating. The opposite of Personal attack is Personal praise, where a person's qualities and virtues are glorified instead of their work/policy/contribution. It is very common to see politicians engaging in personal attacks towards the leaders of

\footnotetext{
${ }^{4}$ https://en.wikipedia.org/wiki/ Propaganda_techniques
}

other parties, and highly praising their own party leaders.

4. Repetition: Ad Nauseam or Repetition is a way of introducing bias by repeating an idea, question, or a slogan in an attempt to subconsciously establish that as the truth for the reader.

5. Appeal to Audience: This is a technique where the audience is persuaded or won over by appealing to various aspects of their lives such as their fears, beliefs and interests. This technique most commonly occurs in the following ways:

- Appeal to fear: This happens when media or a politician seeks to build support of the audience by instilling panic and fear in them, usually about the outcome of not following the suggested course of action.

- Appeal to beliefs and prejudices: This happens when media or a politician seeks to build a connection with the audience by bringing up shared beliefs and prejudices, and using them to influence the opinion of the audience.

This often happens when politicians want to alienate or create a distance between the audience and any other party, by portraying it as an "outside entity" which does not fit in their shared beliefs and opinions.

- Appeal to shared identity: This happens when media or a politician appears to be a "part" of some aspect of the identity of the intended audience, and highlights the image of them as a part of that shared identity.

Unlike beliefs, these aspects of identities are more concrete. For example, a politician dressing up like a farmer and sowing some crops while campaigning and appealing to farmers.

- Appeal by making promises: This includes trying to persuade a specific set of audience by making promises which benefit them.

6. Bandwagon: This is a technique where the audience is persuaded to follow a particular path by presenting it as the most favourable 
path which all others are taking. This is done by assuring good results, such as guaranteed victory in the elections.

\section{Black and White Fallacy/Rhetorical Ques-}

tions: Black and White fallacy involves presenting the audience with only two choices, while clearly showing only one of them as the most suitable choice. In case of political news, this is also seen in the form of rhetoric yes/no questions, where only one of the answers seems logically correct in that particular context.

Another similar way of using questions to persuade someone is called Hypophora, where the audience is presented with a question, and the speakers themselves give a seemingly correct answer along with an explanation, without giving the audience much time to contemplate. This is done to subconsciously make the audience accept the proposed answer as the correct one without questioning it further.

8. Intentional Vagueness: Intentionally presenting the audience with vague information, unstated assumptions or unsupported claims falls under this category. This is often done to make the audience draw certain expected conclusions, which would have been difficult if they knew the whole scenario clearly.

For example, there has been study conducted by Kameswari et al. (2020) which shows how introducing vagueness in the form of presupposition contributes to bias in an article.

9. Oversimplification: The idea behind this technique is to oversimplify the implications of following/not following a course of action and showing that it leads to some nonacceptable outcome.

This is similar to a philosophical argument called Reductio ad absurdum, where one attempts to establish a claim by showing that the opposite scenario would lead to absurdity or contradiction.

These ways are often used to persuade the audience to support a presented idea, person or a political party without directly asking them to do so.

10. Whataboutism: This is a logical fallacy used to discredit an opponent's claim by deflecting it to something else. This mostly happens in the scenarios where one is questioned about his/her own actions. There have been studies by Islam (2018) and Dykstra et al. (2020) which show how politicians use whataboutism to dodge questions.

For instance, when the former US President Donald Trump was asked about his opinion on whether Virginia should keep a statue of Confederate general Robert E. Lee, he did not give a straightforward answer but resorted to this technique by asking, "What about other statues of famous Americans?"

\subsection{Annotation Guidelines and Procedure}

Each article was already annotated for the polarity of the bias ( -1 for negative, 1 for positive and 0 for unbiased) in the corpus we created from the corpus of Gangula et al. (2019). We additionally had the information of the newspaper each article was taken from. There were 3 newspapers from which the articles were taken - Eenadu ${ }^{6}$, Andhrajyothy ${ }^{7}$ and Sakshi ${ }^{8}$.

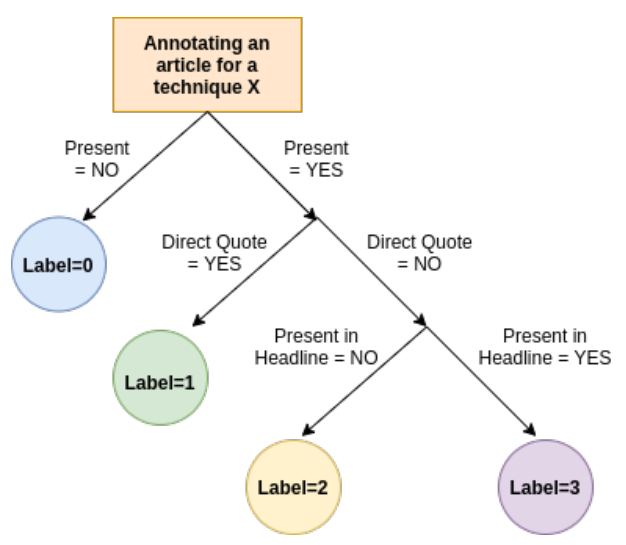

Figure 1: Guidelines for Fine Grained Annotation

Each article was annotated for the presence of the 10 techniques described in Section 3.1. Instead of binary annotation ( 0 or 1 for the presence or absence of a technique), we decided to annotate at a more fine grained level. This was done to capture whether a particular technique was directly quoted, or introduced indirectly while reporting. This kind of indirect incorporation of bias by media while reporting is known as Spin, which was found to

\footnotetext{
${ }^{5}$ https://time.com/4941771/ donald-trump-whataboutism-rhetoric/

${ }^{6}$ https: / / www. eenadu. net

${ }^{7}$ https: / / www . andhrajyothy. com

${ }^{8}$ https: / / www.sakshi.com
} 


\begin{tabular}{|c|c|c|c|c|c|c|c|}
\hline Technique & $\begin{array}{l}\text { Occurrences in } \\
\text { negatively biased } \\
\text { articles }\end{array}$ & $\begin{array}{l}\text { Occurrences in } \\
\text { positively biased } \\
\text { articles }\end{array}$ & $\begin{array}{l}\text { Occurrences in } \\
\text { neutral articles }\end{array}$ & $\begin{array}{l}\text { Direct occurrences } \\
\text { (Label=1) }\end{array}$ & $\begin{array}{l}\text { Indirect occurrences } \\
\text { (Label=2) }\end{array}$ & $\begin{array}{l}\text { Headline occurrences } \\
\text { (Label=3) }\end{array}$ & $\begin{array}{l}\text { Total } \\
\text { occurrences }\end{array}$ \\
\hline Cultural/Identity Bias & 110 & 40 & 8 & 140 & 10 & 8 & 158 \\
\hline Amplification & 285 & 75 & 5 & 245 & 50 & 69 & 365 \\
\hline Personal Targeting & 325 & 95 & 2 & 305 & 20 & 99 & 422 \\
\hline Repetition & 75 & 15 & 4 & 35 & 55 & 4 & 94 \\
\hline Appeal to audience & 165 & 55 & 17 & 210 & 13 & 14 & 237 \\
\hline Bandwagon & 125 & 35 & 4 & 150 & 10 & 4 & 164 \\
\hline $\begin{array}{l}\text { Black and White Fallacy/ } \\
\text { Rhetorical Questions }\end{array}$ & 145 & 25 & 19 & 150 & 15 & 24 & 189 \\
\hline Intentional Vagueness & 275 & 75 & 8 & 205 & 50 & 99 & 358 \\
\hline Oversimplification & 55 & 11 & 5 & 45 & 12 & 14 & 71 \\
\hline Whataboutism & 120 & 15 & 11 & 120 & 15 & 11 & 146 \\
\hline
\end{tabular}

Table 2: Statistics of the number of occurrences of each technique in the dataset

be a frequently occurring phenomena employed to twist the facts and influence public opinion (Mullainathan and Shleifer (2002), Burke (2008)).

For each technique, the annotator had to label 0 if it was not present anywhere in the article, 1 if it was quoted directly in any part of the article, 2 if it was indirectly incorporated in the article, and 3 if it was indirectly incorporated and present in the headline of the article. These guidelines are shown in a hierarchical decision tree format for clear understanding in Figure 1. Each article was annotated by 2 annotators, and the inter-annotator agreement was found to be 0.715 .

\subsection{Statistics}

The statistics of the dataset are presented in Table 2. We observe that the direct occurrences are higher than indirect and headline occurrences for most of the techniques. Personal targeting is the most frequently used technique followed by amplification and intentional vagueness. Oversimplification and repetition are the least frequent techniques in the dataset.

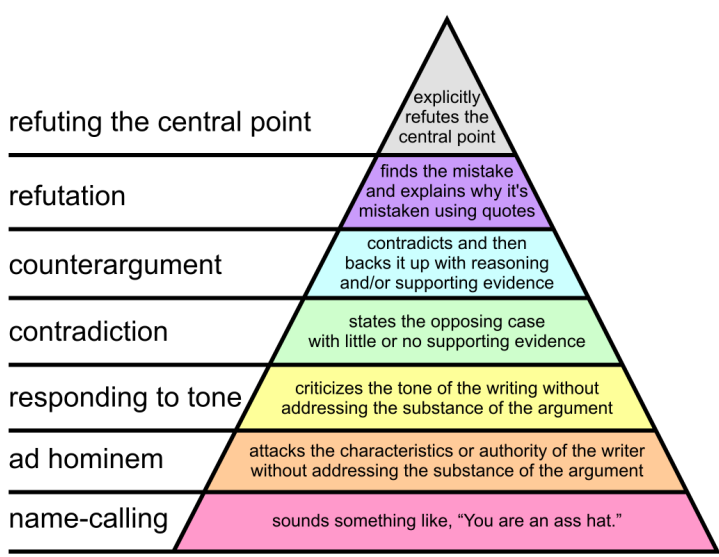

Figure 2: Graham's Hierarchy of Disagreement

\section{Sentiment Based Ranking}

Our first step towards quantifying the magnitude or severity of the political bias in an article is to create a ranking of the techniques mentioned in 3.1 based on how much they contribute to creation of bias in a text. This was inspired by Graham's Hierarchy of Disagreement ${ }^{9}$ which classifies disagreement, particularly on online platforms, into 7 levels as shown in Figure 2.

The least level of disagreement was Name calling, where a person just says repulsive things about another person or group of people. On the other end, the highest level of disagreement was Refuting the central point of an argument.

We claim that the sentiment of an article can be a useful indicator of the severity of political bias, since an unbiased article contains only objective information devoid of positive or negative sentiment. Based on this claim, we calculate the sentiment of each news article as the average of its sentence-level sentiment values $(-1,0,1)$.

\subsection{Model}

For the purpose of sentiment analysis in Telugu, we use IndicBERT (Kakwani et al., Kunchukuttan et al.) which is a multilingual NLU model pretrained on 12 Indian languages and evaluated on IndicGLUE. The model used is ALBERT (Lan et al., 2019), a light-weight, compact version of BERT with fewer parameters. After generating embeddings for each sentence using the model, we follow the same approach as Kunchukuttan et al. (2020) and use a KNN classifier with $\mathrm{k}=4$ for sentiment classification task. This performed with an accuracy of 0.52 and F1 score of 0.54 on ACTSA News dataset (Mukku and Mamidi, 2017), which

\footnotetext{
${ }^{9}$ http: //www.paulgraham.com/disagree. html
} 
is comparable to current state-of-the-art text classification performance in Telugu.

\begin{tabular}{lc}
\hline Technique & $\begin{array}{c}\text { Avg Article } \\
\text { Sentiment }\end{array}$ \\
\hline Personal Targeting & 0.169 \\
\hline Amplification & 0.135 \\
\hline Intentional Vagueness & 0.122 \\
\hline Bandwagon & 0.113 \\
\hline $\begin{array}{l}\text { Black and White Fallacy/ } \\
\text { Rhetorical Questions }\end{array}$ & 0.093 \\
\hline Appeal to Audience & 0.090 \\
\hline Whataboutism & 0.078 \\
\hline Cultural/Identity Bias & 0.073 \\
\hline Repetition & 0.059 \\
\hline Oversimplification & 0.042 \\
\hline
\end{tabular}

Table 3: Average values of the sentiment scores of articles containing each technique in descending order

\subsection{Results}

Using the sentiment values for each article in the annotated dataset, we try to compute the average sentiment value corresponding to each political bias technique. Since our primary interest is to see how much a technique can shift the article from neutrality, we consider only the magnitudes of the sentiment values of each articles. We collect all the articles which contain a particular technique, and calculate the sentiment score of the technique as the average of all the absolute values of the article sentiments.

According to initial claim, the higher the sentiment value of a technique, the higher is its contribution towards creating political bias in an article. The sentiment value for each technique is shown in Table 3. Personal Targeting has the highest value, which can be supported by the fact that attacking or praising an individual/party instead of focusing on policies/actions is the most direct way of introducing bias. It can also be observed from Table 2 that Personal Targeting was observed in around $84 \%$ of the articles in our dataset, making it the most common and frequently used technique in Political bias. Figure 3 shows the sentiment ranking of the techniques in a graph.

\section{Schema Validation}

To test the reliability of the schema proposed in Section 4.2, we tested it on a bias magnitude comparison task against the human annotation results. For this purpose, we created a test dataset consisting of 100 article pairs. For each pair, the task was to identify the more biased article of the two. All the articles were taken from the political news domain. For human annotation, we had the annotation done by two annotators who had no idea about the schema. We removed the articles on which there was a disagreement between the two annotators. We were left with 92 articles.

Then we gave the test pairs to two other annotators who had the list of the 10 techniques along the definition, explanation and examples. Their task was to identify all the techniques present in the two articles given in each article pair. Then they were instructed to give bias scores for each article by adding the scores of all the techniques from Table 3. In a given pair, the article having a higher score was labelled as the one having a higher magnitude of bias.

If both the articles in a pair had same bias score but at least one non-common label, the comparison was to be done based on the non-common label. If two articles had the same score and same labels, we asked the annotators to mark the shorter of the two articles as the more biased one.

We found that 66 out of 92 times, we were able to correctly identify the more biased article using our ranking. This gives us confidence that the ordering of techniques done through our ranking has a strong correlation with the relative contribution of each technique towards political bias in an article.

\section{Conclusion and Future Work}

This paper explores the creation of a novel annotation schema which captures the nuances of political bias in a fine grained manner. We propose a sentiment analysis based ranking of 10 political bias techniques, and a validation study to show that this ranking corresponds to the relative contribution of each of these techniques towards political bias. We also contribute a fully annotated dataset- $\mathrm{PoBiCo}-$ 21 , containing 500 articles annotated using this schema $^{10}$. The fine grained annotation can also be used for making other interesting observations from political news data such as identifying media houses which often use biased/misleading headlines, mining frequently co-occuring techniques of political bias, etc. 


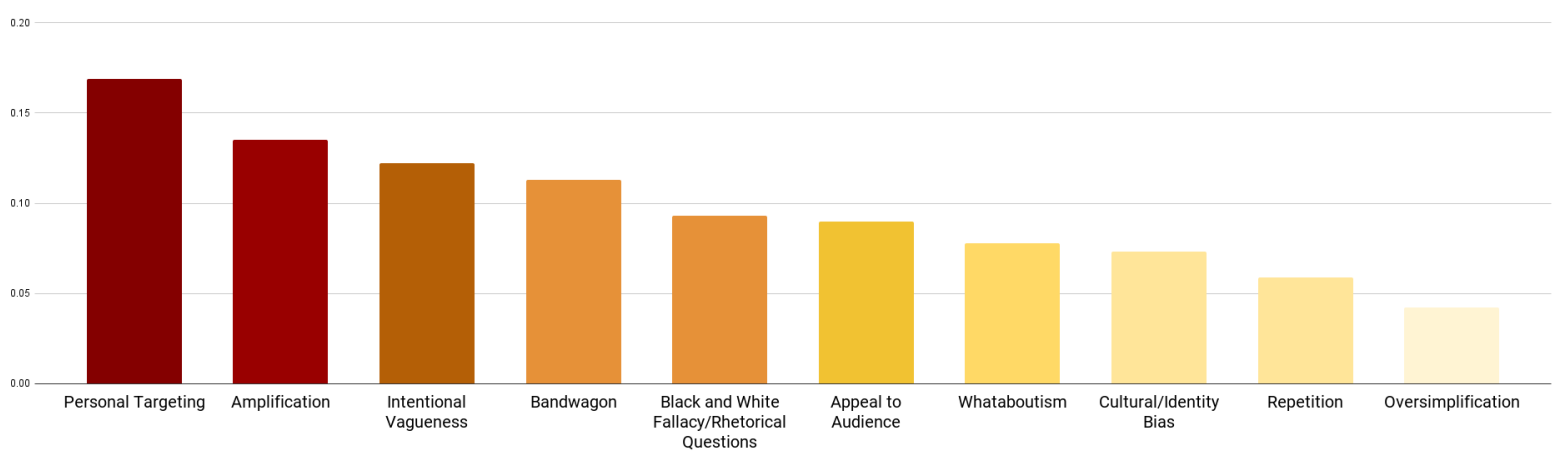

Figure 3: Relative Scores of each technique based on their article sentiment ranking
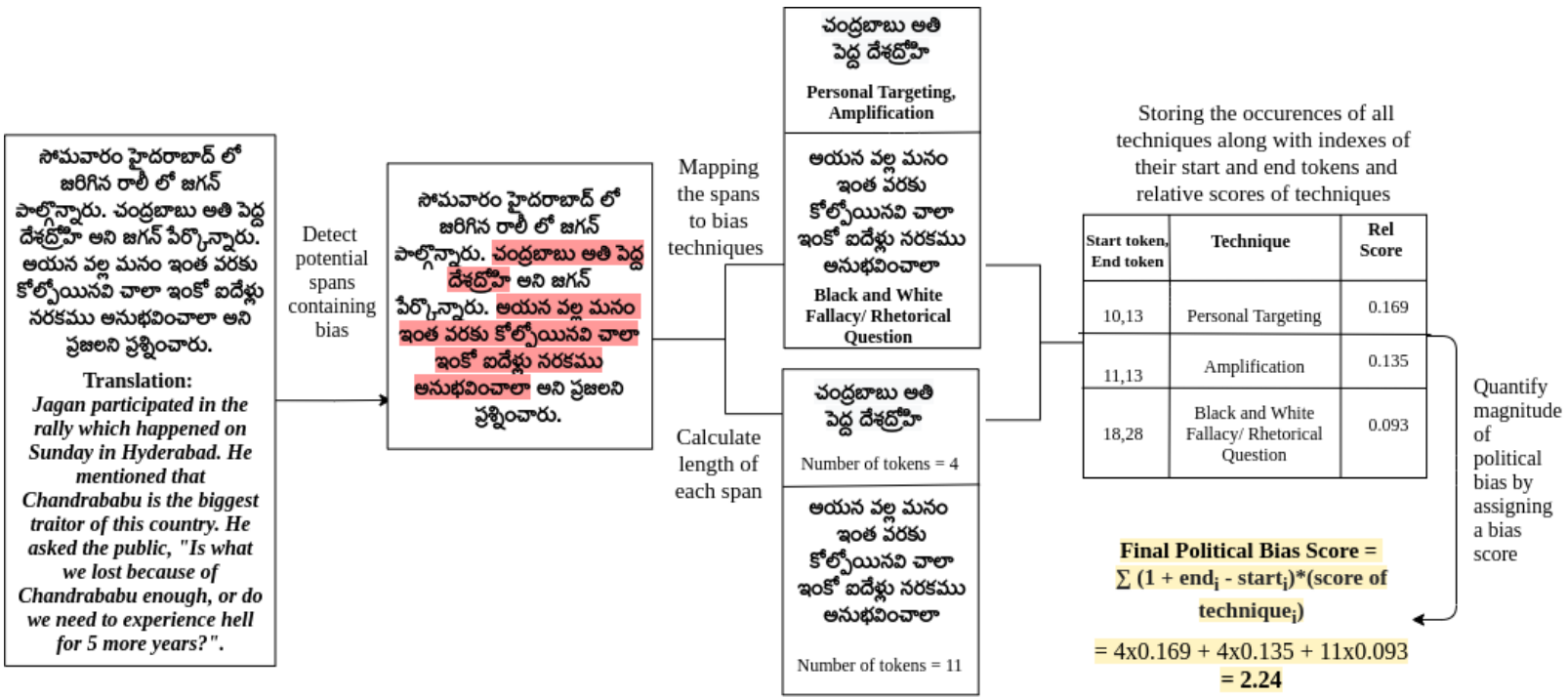

Figure 4: Process workflow of the proposed system of Bias Scoring

\subsection{Proposed Direction for Future Research}

In the future, we can create a end-to-end bias scoring system which takes an article as an input, tokenises it, detects all potential spans of text conatining political bias, assigns the relevant political bias techniques to each span, and computes the final score as the summation of each span length multiplied by the relative sentiment scores of the techniques present in that span. This is shown in a step-by-step manner with an example in Figure 4.

Detection of bias spans can be treated as a standard sequence labelling problem. With good amount of labelled data, we can use Neural Transformer architectures like BERT (Devlin et al., 2018), or its variants like RoBERTa (Liu et al., 2019), ALBERT (Lan et al., 2019) etc. Once that is done, we can do a one-to-many mapping from

\footnotetext{
${ }^{10}$ https://github. com/valalithak/ $\mathrm{POBiCO}-21$
}

spans to the political bias techniques and then compute the final score of the article.

With the help of this system, a reader can not only quantify and compare the magnitude of political bias of several articles, but also identify the techniques which are being used either directly (by the politicians) or indirectly (by the media) to create bias.

\section{References}

Dylan Bourgeois, Jérémie Rappaz, and Karl Aberer. 2019. Selection bias in news coverage: Learning it, fighting it. CoRR, abs/1904.07536.

Jeremy Burke. 2008. Primetime spin: Media bias and belief confirming information. Journal of Economics \& Management Strategy, 17(3):633-665.

André Ferreira Cruz, Gil Rocha, and Henrique Lopes Cardoso. 2020. On document representations for detection of biased news articles. In Proceedings of 
the 35th Annual ACM Symposium on Applied Computing, pages 892-899.

Jacob Devlin, Ming-Wei Chang, Kenton Lee, and Kristina Toutanova. 2018. Bert: Pre-training of deep bidirectional transformers for language understanding. arXiv preprint arXiv:1810.04805.

Allan Dykstra et al. 2020. The rhetoric of "whataboutism" in american journalism and political identity. Res Rhetorica, 7(2):2-16.

Lisa Fan, Marshall White, Eva Sharma, Ruisi Su, Prafulla Kumar Choubey, Ruihong Huang, and Lu Wang. 2019. In plain sight: Media bias through the lens of factual reporting. arXiv preprint arXiv:1909.02670.

Rama Rohit Reddy Gangula, Suma Reddy Duggenpudi, and Radhika Mamidi. 2019. Detecting political bias in news articles using headline attention. In Proceedings of the 2019 ACL Workshop BlackboxNLP: Analyzing and Interpreting Neural Networks for NLP, pages 77-84, Florence, Italy. Association for Computational Linguistics.

Mark R Grandstaff. 2006. Propaganda and mass persuasion: A historical encyclopedia, 1500 to the present. The Journal of Military History, 70(2):568569.

Edward S Herman and Noam Chomsky. 2010. Manufacturing consent: The political economy of the mass media. Random House.

Shahinul Islam. 2018. Bangladeshi politicians, the people, and whataboutism. A Journal of English Studies, 9 .

Maojin Jiang and Shlomo Argamon. 2008. Exploiting subjectivity analysis in blogs to improve political leaning categorization. In Proceedings of the 31 st annual international ACM SIGIR conference on Research and development in information retrieval, pages 725-726.

Divyanshu Kakwani, Anoop Kunchukuttan, Satish Golla, Gokul N.C., Avik Bhattacharyya, Mitesh M. Khapra, and Pratyush Kumar. 2020. IndicNLPSuite: Monolingual Corpora, Evaluation Benchmarks and Pre-trained Multilingual Language Models for Indian Languages. In Findings of EMNLP.

Lalitha Kameswari, Dama Sravani, and Radhika Mamidi. 2020. Enhancing bias detection in political news using pragmatic presupposition. In Proceedings of the Eighth International Workshop on Natural Language Processing for Social Media, pages $1-6$.

Anoop Kunchukuttan, Divyanshu Kakwani, Satish Golla, Avik Bhattacharyya, Mitesh M Khapra, Pratyush Kumar, et al. 2020. Ai4bharat-indicnlp corpus: Monolingual corpora and word embeddings for indic languages. arXiv preprint arXiv:2005.00085.
Zhenzhong Lan, Mingda Chen, Sebastian Goodman, Kevin Gimpel, Piyush Sharma, and Radu Soricut. 2019. ALBERT: A lite BERT for selfsupervised learning of language representations. CoRR, abs/1909.11942.

Yinhan Liu, Myle Ott, Naman Goyal, Jingfei Du, Mandar Joshi, Danqi Chen, Omer Levy, Mike Lewis, Luke Zettlemoyer, and Veselin Stoyanov. 2019. Roberta: A robustly optimized bert pretraining approach. arXiv preprint arXiv:1907.11692.

Sandeep Sricharan Mukku and Radhika Mamidi. 2017. Actsa: Annotated corpus for telugu sentiment analysis. In Proceedings of the First Workshop on Building Linguistically Generalizable NLP Systems, pages 54-58.

Sendhil Mullainathan and Andrei Shleifer. 2002. Media bias. Technical report, National Bureau of Economic Research.

Zohre Sivandi Nasab and Hamid Reza Dowlatabadi. 2015. An investigation into rohani's meeting coverage in two english daily newspapers with a critical discourse analysis perspective: Tehran times vs. los angeles times. Theory and Practice in Language Studies, 5(10):2131-2137.

Daniel Xiaodan Zhou, Paul Resnick, and Qiaozhu Mei. 2011. Classifying the political leaning of news articles and users from user votes. In Proceedings of the international AAAI conference on web and social media, volume 5 . 